\title{
A PREPARATIVE 2D-CHROMATOGRAPHY METHOD FOR COMPOUND-SPECIFIC RADIOCARBON ANALYSIS OF DICARBOXYLIC ACIDS IN AEROSOLS
}

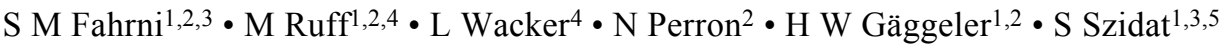 \\ ABSTRACT. There is a great scientific demand for an assessment of the sources and formation processes of atmospheric \\ carbonaceous aerosols since they strongly influence the global radiation balance and affect public health. Much attention in \\ atmospheric studies has been paid to dicarboxylic acids (DCAs) due to their abundance at substantially different sites and \\ their potential influence on cloud formation processes. Nevertheless, sources of oxalic acid (HOOCCOOH) and other DCAs \\ are not well understood yet. In order to quantify contributions of fossil and non-fossil sources, a method for the preparative \\ separation of oxalic acid and other DCAs from aerosols for compound-specific radiocarbon analysis (CSRA) has been devel- \\ oped. This method consists of a water extraction of aerosols collected on quartz-fiber filters followed by 2 consecutive liquid \\ chromatography (LC) steps on different chromatography columns (2D-chromatography). Through the use of aqueous, com- \\ pletely non-organic eluents and single injections into liquid chromatography, low blank levels are achieved with total oxalic \\ acid recoveries of up to $66 \%$. Upon separation, ${ }^{14} \mathrm{C}$ measurements of small samples (containing typically $10-20 \mu \mathrm{g}$ carbon) \\ are conducted at the gas ion source of the $200 \mathrm{kV}$ accelerator mass spectrometry facility MICADAS. The method is verified \\ with processed reference materials, artificial mixtures of oxalic acid with typical matrix components, and a standard addition \\ of ambient aerosols. Two exemplary field samples show dominant non-fossil sources of oxalic acid.
}

\section{INTRODUCTION}

Much effort has been put into the investigation of dicarboxylic acids (DCAs) in carbonaceous aerosols. DCAs are found at rural (Legrand et al. 2007), urban (Kawamura and Ikushima 1993), and marine sites (Kawamura and Usukura 1993), which raises questions about their sources, precursors, and formation processes. Moreover, DCAs may play a role as cloud condensation nuclei (Hori et al. 2003). Consistently, the main dicarboxylic acid found in aerosols is oxalic acid (mainly in its deprotonated form, oxalate) with concentrations of tens to hundreds of $\mathrm{ng} / \mathrm{m}^{3}$ air (Kawamura and Usukura 1993; Hsieh et al. 2007; Legrand et al. 2007). Recent studies have revealed a strong diurnal correlation of ozone with the oxalic acid content of aerosols (Fisseha et al. 2009) and many other investigations concluded that this low-molecular weight DCA is produced mainly through oxidation of volatile organic compounds (VOCs). A brief summary of proposed precursors and formation processes of $\mathrm{C}_{2}$ to $\mathrm{C}_{6}$ diacids is given in Legrand et al. (2007).

A powerful tool for source apportionment of aerosols is provided by radiocarbon measurements. Aerosol components from non-fossil sources show a modern ${ }^{14} \mathrm{C}$ content while components from fossil sources are depleted in ${ }^{14} \mathrm{C}$. A well-established way of analyzing aerosols using ${ }^{14} \mathrm{C}$ involves a thermal separation and combustion of organic carbon (OC) and elemental carbon (EC) (Szidat et al. 2004, 2009). For compound-specific radiocarbon analysis (CSRA), however, a good chemical separation of the compounds of interest is needed prior to ${ }^{14} \mathrm{C}$ measurements. CSRA measurements of $\mathrm{C}_{16}$ fatty acids and several pooled fractions of $\mathrm{C}_{18}$ to $\mathrm{C}_{32}$ fatty acids in aerosols were achieved by Matsumoto et al. (2001) using gas chromatography (GC). Mandalakis et al. (2005) performed CSRA measurements of atmospheric polycyclic aromatic hydrocarbons (PAHs). The main difficulty revealed in those studies was the low abundance of the compounds of interest. In order to obtain sufficient carbon masses for accelerator mass spectrometry (AMS) measurements, most iso-

\footnotetext{
${ }^{1}$ Department of Chemistry and Biochemistry, University of Bern, Bern, Switzerland.

${ }^{2}$ Paul Scherrer Institut, Villigen, Switzerland.

${ }^{3}$ Oeschger Centre for Climate Change Research, University of Bern, Bern, Switzerland.

${ }^{4}$ Ion Beam Physics, ETH Hönggerberg, Zurich, Switzerland.

${ }^{5}$ Corresponding author. Email: szidat@iac.unibe.ch.
}

(C) 2010 by the Arizona Board of Regents on behalf of the University of Arizona Proceedings of the 20th International Radiocarbon Conference, edited by A J T Jull RADIOCARBON, Vol 52, Nr 2-3, 2010, p 752-760 
lated compound fractions had to be pooled. It is therefore evident that CSRA is restricted to the most abundant aerosol compounds and an AMS facility capable of handling the resulting very small samples is needed.

For gaseous routine measurements at MICADAS (Synal et al. 2004; Ruff et al. 2007, 2010), amounts of $10 \mu \mathrm{g} \mathrm{C}$ are desirable. However, it is possible to measure amounts down to $2 \mu \mathrm{g} \mathrm{C}$. Samples in this size range are very prone to contamination; therefore, it is crucial to minimize blank levels in order to obtain reliable data from ${ }^{14} \mathrm{C}$ measurements. Another important prerequisite for accurate ${ }^{14} \mathrm{C}$ results from compound-specific ${ }^{14} \mathrm{C}$ measurements is the good separation of the compounds of interest from other organic compounds. In contrast to Matsumoto et al. (2001) and Mandalakis et al. (2005) who used gas chromatography (GC) as a separation method, we decided to use liquid chromatography (LC). This allows separating very polar and non-volatile compounds like oxalic acid without derivatization, and enough material for AMS measurements can be obtained from single injections. In the case of aerosol studies, however, very high matrix contents need to be eliminated to obtain single compounds for ${ }^{14} \mathrm{C}$ measurements. The main problem with high matrix concentrations is overloading of analytical chromatography columns, resulting in insufficient separation. Especially for single injections of aerosol samples into LC, sufficient separation is a nontrivial task since sample amounts lie in the high microgram to low milligram range, while widespread LC systems and columns are designed for very low analyte concentrations. Moreover, most detection methods in LC are insensitive to certain compounds and contaminants from the sample matrix that may thus be introduced into the supposedly purified fraction without being noticed. A good separation should, however, not be obtained at the cost of yield due to the low contents of analytes that are available.

In this article, we present a novel 2D-chromatography procedure for the preparative separation of DCAs in ambient aerosols. A verification of the method with reference materials is shown and the importance of blank values for very small samples is discussed.

\section{METHODS}

\section{Aerosol Sampling and Sample Treatment}

Aerosol samples used in this work were collected with HiVol samplers in the $\mathrm{PM}_{10}$ mode (i.e. ambient air was sucked through prebaked quartz-fiber filters at a high flow rate and particulate matter with an aerodynamic diameter of $<10 \mu \mathrm{m}$ was acquired). Aerosol samples were collected on pre-baked quartz-fiber filters and stored in aluminium foil and air-tight plastic bags at $-20{ }^{\circ} \mathrm{C}$ until analysis. Filter samples were exposed to ambient temperatures for $48 \mathrm{hr}$ during transportation. Before taking them out of the plastic bag, the filters were allowed to reach room temperature to prevent condensation.

The sample "Gothenburg" consisted of 5 pooled aerosol filters collected $30 \mathrm{~m}$ above ground in downtown Gothenburg, Sweden $\left(57^{\circ} 42^{\prime} \mathrm{N}, 11^{\circ} 57^{\prime} \mathrm{E}\right)$ from 11 February to 4 March 2005. Filters from the whole time period were combined to obtain a time-averaged value for the analysis. Filter material corresponding to $1180 \mathrm{~m}^{3}$ air was used for sample preparation. More information on the Gothenburg filters is given in Szidat et al. (2009).

The sample "Ispra" was collected at a rural site in Ispra, northern Italy, near Lake Maggiore $\left(45^{\circ} 49^{\prime} \mathrm{N}, 8^{\circ} 37^{\prime} \mathrm{E}\right)$. Sampling was performed $2 \mathrm{~m}$ above ground from $15-19$ September 2008. Two samples of the same filter were analyzed to check the consistency of the separation procedure. Two aliquots corresponding to about $850 \mathrm{~m}^{3}$ each were prepared. 


\section{Water Extraction of Aerosol Filters}

The procedure described in the following paragraphs is illustrated in Figure 1 for clarity. For extraction, the filter material was put into a rinsed and dried $15-\mathrm{mL}$ polypropylene tube (Sarstedt, Germany) and covered with ultrapure water $(18.2 \mathrm{M} \Omega / \mathrm{cm})$. The tube was vortexed, sonicated for $10 \mathrm{~min}$, and vortexed again. The plunger of a rinsed 5-mL syringe (Codan Medical ApS, Denmark) was removed and the brownish filter extract and the filter itself were filled into the syringe barrel. The extract was pressed through a syringe filter $(25 \mathrm{~mm}$ OD, $0.45-\mu \mathrm{m}$ pore, Semadeni, Switzerland) in order to remove filter debris and suspended particles. The filtered extract was collected in $5-\mathrm{mL}$ PFA vials (Savillex, USA). Filter material was pressed out completely and thereafter transferred to the plastic tube again. Extraction and filtration steps were repeated as described above. Upon pressing out the filter material into PFA vials after the last extraction step, $0.5 \mathrm{~mL}$ of ultrapure water were poured through the used syringe and filter to flush out the remaining extract therein. The overall extraction yield was determined by weighing the initial and final masses of water during the procedure. Two consecutive extraction steps have led to a yield of $>97 \%$, which was considered to be sufficient. The collected extracts were concentrated overnight under a gentle $\mathrm{N}_{2}$ stream at $40{ }^{\circ} \mathrm{C}$ in a laminar flow box to give a final volume of $50 \mu \mathrm{L}$.

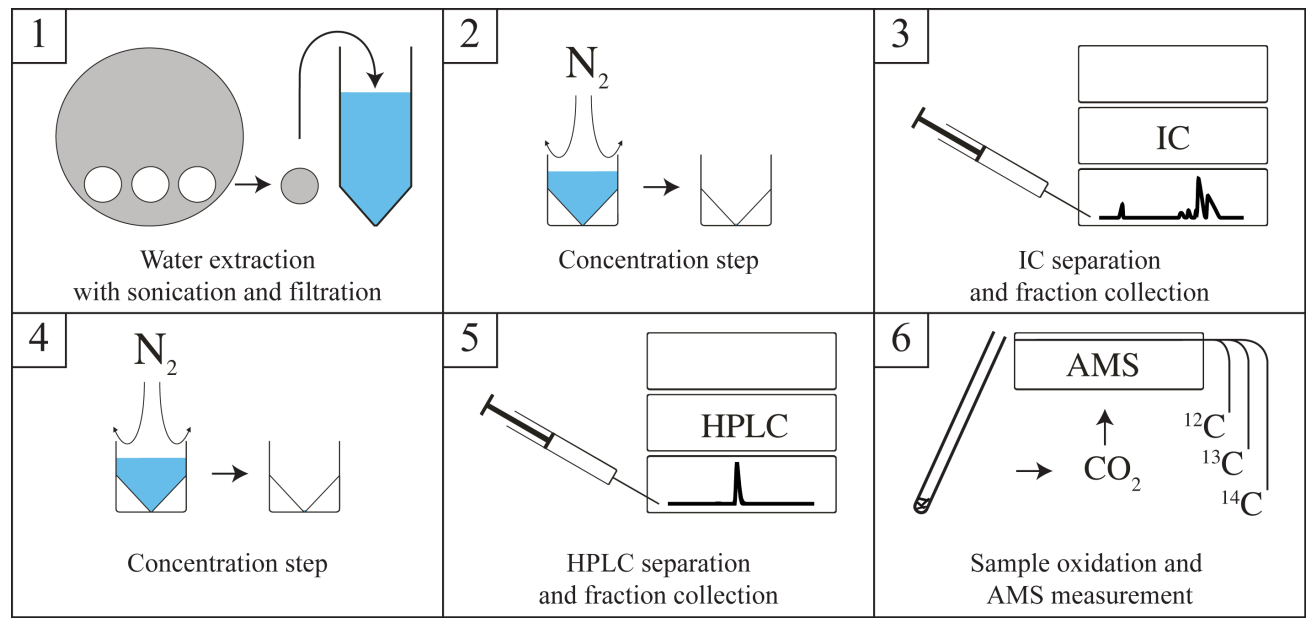

Figure 1 Flow chart of the procedure described for air filter samples

\section{Preparative Liquid Chromatography Separation}

Concentrated extracts originating from the same samples were combined and loaded into a $100-\mu \mathrm{L}$ syringe (Hamilton, Switzerland) for injection into the ion chromatograph (IC). Our IC system (DX120, Dionex, USA) consists of an actuated 6-port valve (9750E, Rheodyne, USA) with a 100- $\mu \mathrm{L}$ sample loop, a high-capacity IonPac AS11-HC $4 \times 250 \mathrm{~mm}$ analytical column, an ASRS 300 suppressor, a reagent-free controller RFC-30, and a standard conductivity detector CDM-3 (all from Dionex, USA). The suppressor was supplied with an external water flow in order to allow a preparative separation. Ultrapure degassed water was used as an eluent and the $\mathrm{KOH}$ concentration was controlled through the RFC-30 device. After 5 min with the initial eluent composition of $5 \mathrm{mM}$ $\mathrm{KOH}$, a linear gradient to $50 \mathrm{mM} \mathrm{KOH}$ was applied for $40 \mathrm{~min}$. The flow was kept at $1 \mathrm{~mL} / \mathrm{min}$. The oxalate fraction was collected in a PFA vial and concentrated to a volume of $25 \mu \mathrm{L}$ for injection into HPLC to prevent volume overloading. The HPLC system used (UltiMate 3000, Dionex, USA) con- 
sists of a degassing unit, a quaternary pump, and a column oven compartment. Samples were loaded into a 50- $\mu \mathrm{L}$ sample loop and separation was performed on an Acclaim OA $4 \times 250 \mathrm{~mm}$ analytical column. The eluent consisted of ultrapure water with $5 \mathrm{mM} \mathrm{HCl}$ (AR grade, Merck, Germany) and separation was performed in an isocratic run of $5 \mathrm{~min}$ with a flow of $1 \mathrm{~mL} / \mathrm{min}$. Analytes were detected with an evaporative light-scattering detector (ELS 2100 Ice, Polymer Labs, USA), which was operated in a split manner: $90 \%$ of the flow was used for fraction collection, $10 \%$ for the ELS detector. Fraction collection was automated using a Foxy 200 fraction collector (Isco, USA) with PFA vials. Collected fractions were then concentrated and prepared for combustion of the organic compounds.

\section{Sample Combustion for Gaseous AMS Measurements}

After separation, the concentrated HPLC eluate was transferred to a quartz tube ( $3.5 \mathrm{~mm}$ ID, length $15 \mathrm{~cm}$ ) filled with about $100 \mathrm{mg}$ of cupric oxide (both prebaked to remove possible contaminations). Water was removed at a vacuum line, the evacuated tube was sealed after complete water removal and heated to $950{ }^{\circ} \mathrm{C}$ for $\sim 2 \mathrm{hr}$ in order to oxidize the organic compounds. Without any further processing, the gaseous ${ }^{14} \mathrm{C}$ measurement was conducted directly after oxidation (Fahrni et al. 2010). Ampoules with $\mathrm{CO}_{2}$ were handled with the cracker system described in Ruff et al. (2007). The released $\mathrm{CO}_{2}$ was flushed into a gas syringe by means of helium and the resulting $\mathrm{CO}_{2} / \mathrm{He}$ mixture was pressed into the gas ion source by a stepping motor. All of the presented ${ }^{14} \mathrm{C}$ results were obtained with the small AMS facility MICADAS (Synal et al. 2004). Details on gas measurements and handling of standards and blanks can be found in Ruff et al. (2010) and Fahrni et al. (2010).

\section{Contamination Precautions}

Cleaning procedures were important in order to minimize carbon contaminations. All samples were treated in laminar flow boxes. All plastic equipment was rinsed with ultrapure water at least 3 times. PFA vials were cleaned mechanically with precision wipes, rinsed with ultrapure water, and then sonicated in a basic and acidic washing step prior to thorough rinsing with ultrapure water. Wet equipment was allowed to dry in the laminar flow box. Chromatography eluent solutions were prepared directly before separation and the syringe was rinsed thoroughly before and between samples. Due to the occasional heavy overloading, chromatography columns had to be cleaned to avoid cross contamination of samples. Cleaning was achieved by running 1-2 blank samples (ultrapure water) until chromatograms reached typical blank values.

\section{Method Verification with Artificial Samples}

For blank assessment and verification of the described method, artificial samples of IAEA C7 and NIST OxII reference materials (both oxalic acid with 49.5 and $134.1 \mathrm{pMC}$, respectively) were processed according to the protocol described in the previous paragraph. A sulfate concentration of $1000 \mathrm{ppm}$ was maintained in all sample solutions to simulate the high sulfate content in ambient aerosol samples. Three types of artificial samples were produced:

1. C7 or OxII was dissolved in a 1000-ppm sulfate solution $(1 \mathrm{mg} / \mathrm{mL}$ carbon) made from ultrapure water and $\left(\mathrm{NH}_{4}\right)_{2} \mathrm{SO}_{4}$ (Merck, Germany). Volumes of 20 and $50 \mu \mathrm{L}$ of those solutions (corresponding to 20 and $50 \mu \mathrm{g} \mathrm{C}$, respectively) were injected into the IC and processed further according to the described method to investigate column bleeding or any other source of contamination or isotopic fractionation.

2. To evaluate the quality of separation and effects on more realistic, smaller samples, a mixture of a few relevant DCAs (Hsieh et al. 2007) was prepared in the following way: fossil malic, 
maleic, succinic, and malonic acids were mixed to a final concentration of $0.08 \mathrm{mg} / \mathrm{mL}$ carbon per compound with $0.4 \mathrm{mg} / \mathrm{mL}$ carbon from oxalic acid reference material (C7 or OxII) and $1000 \mathrm{ppm}$ sulfate.

3. An oversaturated solution of ${ }^{14} \mathrm{C}$-free humic acids (Fluka, Switzerland) was prepared and filtered through a rinsed syringe-filter $(0.45-\mu \mathrm{m}$ pore $)$ to obtain a saturated solution. Humic acids (HA) were used as a model substance for humic-like substances in atmospheric aerosols (Limbeck et al. 2005). This solution was mixed with the stock solution containing the 4 DCAs, the sulfate, and the $\mathrm{C} 7$ or OxII solution, respectively. Final concentrations were $0.4 \mathrm{mg} / \mathrm{mL}$ C for the oxalic acid reference material, $0.08 \mathrm{mg} / \mathrm{mL}$ C for the 4 dicarboxylic acids, and $0.37 \mathrm{mg} / \mathrm{mL}$ $\mathrm{C}$ for the HA.

Fifty- $\mu \mathrm{L}$ samples ( $20 \mu \mathrm{g} \mathrm{C}$ from oxalic acid) of solutions 2 and 3 were injected into the IC and oxalic acid was isolated and combusted in the described manner to verify the quality of the separation.

\section{Method Verification with a Standard Addition}

As a further test for the robustness of the method, a standard addition was performed. Therefore, the ambient filter sample Ispra was extracted with water. A part of this extract was processed according to the described method and oxalic acid was isolated for combustion and subsequent ${ }^{14} \mathrm{C}$ measurement. The remaining extract was spiked with a known amount of $\mathrm{C} 7$ reference material solution (same oxalic acid concentration as sample extract) and a part of the spiked extract was processed for ${ }^{14} \mathrm{C}$ measurement of the oxalic acid fraction. The rest of the spiked extract was spiked again with $\mathrm{C} 7$ and oxalic acid was isolated for another ${ }^{14} \mathrm{C}$ measurement.

\section{RESULTS AND DISCUSSION}

The goal to obtain $10 \mu \mathrm{g}$ of carbon for a ${ }^{14} \mathrm{C}$ measurement in a single $\mathrm{LC}$ injection leads to a high concentration of sample material. The first injections of aerosol filter extracts on a dedicated HPLC column revealed heavy overloading of the analytical column, which led to insufficient separation and co-elution of substances. Ion chromatography was therefore chosen as a first separation step, as it was found to be more selective, reproducible, and convenient than other techniques while minimizing the potential for contaminations at the same time. The high-capacity anion column allows high loading and the separation of the oxalate fraction upon single injections. Nevertheless, the high loading still impaired the separation and the use of HPLC as a second, consecutive step was chosen to obtain the baseline separation needed for CSRA. This 2D-chromatography approach allows handling of a broad range of samples, makes the method robust, and enables the unambiguous attribution of compounds.

In the presented method, water extraction of aerosols collected on quartz-fiber filters serves as a preseparation as only water-soluble organic carbons (WSOC) and inorganic compounds enter the next step. The water-extracted fraction is then injected into the IC system where cations and neutral compounds are washed off the column and anions are separated through ion exchange. HPLC separation eliminates remaining impurities from the oxalate fraction and the acidic eluent prevents $\mathrm{CO}_{2}$ from being absorbed into the solution. Moreover, other DCAs can only be isolated by an additional HPLC step due to the high matrix content on the IC column. The use of organic chemicals or solvents was avoided in any step of the procedure since even HPLC-grade solvents or high-purity organic acids/ bases for eluents contain impurities that may lead to elevated blanks in ${ }^{14} \mathrm{C}$ measurements. Further precautions in order to reach low contamination levels are described in the experimental section. The total recovery of the chemical separation including $\mathrm{CO}_{2}$ production for AMS measurements was found to be up to $66 \%$. Figure 2 shows the 2 chromatography steps for an ambient aerosol sam- 
ple of $15 \mu \mathrm{g} \mathrm{C}\left(56 \mu \mathrm{g}\right.$ oxalate), which allowed ${ }^{14} \mathrm{C}$ analysis of $10 \mu \mathrm{g} \mathrm{C} \mathrm{(37} \mu \mathrm{g}$ oxalate) after separation. It is important to note that HPLC separation is crucial even if contaminations shown in the HPLC chromatogram seem negligible. Oxalate fractions from IC often showed a yellowish coloration, which could be removed by HPLC. Depending on sample material, higher matrix contents may deteriorate IC separation and make the HPLC separation even more relevant than may become obvious from Figure 2 .

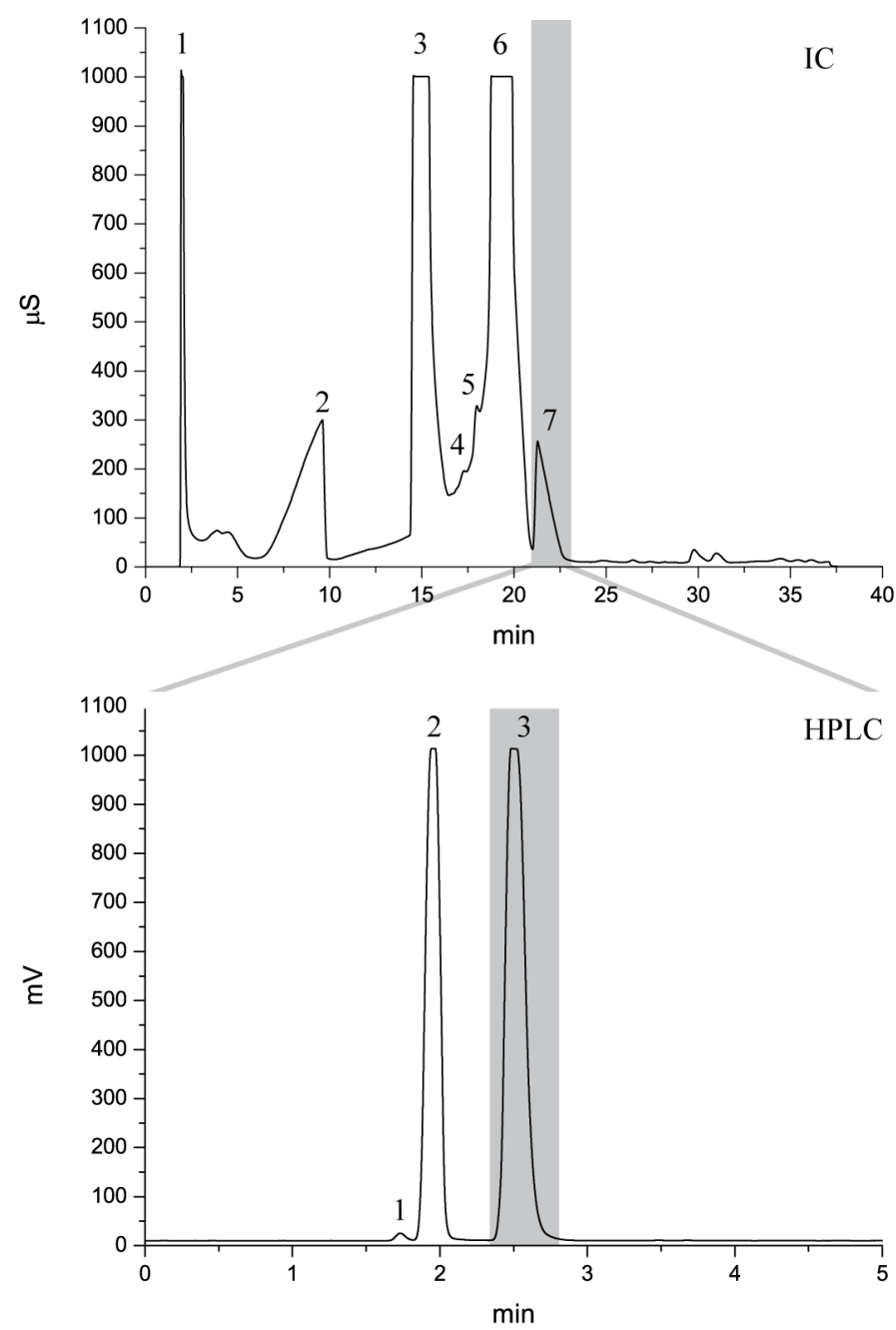

Figure 2 Typical IC and HPLC chromatograms upon single injection of an ambient aerosol extract. Gray bars indicate the collected oxalate fractions. The IC fraction is injected into HPLC, resulting in the lower chromatogram. IC peaks: 1. non-retained analytes; 2 . humic-like substances; 3 . nitrate; 4 . succinate; 5 . malonate and maleate; 6 . sulfate; 7. oxalate. HPLC peaks: 1 . non-retained analytes; 2. sulfate; 3 . oxalate. Detectors reached saturation in the highest peaks, which is the reason for the apparently low noise level and cropped peaks.

Experiments with artificial samples of $\mathrm{C} 7$ and OxII reference materials demonstrated a good separation of oxalic acid from other DCAs and HA. Since the 4 DCAs and the HA were depleted in ${ }^{14} \mathrm{C}$, 
an insufficient separation would have led to lowered ${ }^{14} \mathrm{C}$ values for both $\mathrm{C} 7$ and OxII material. Such a lowering of values was not observed. AMS results are summarized in Table 1. One sample from Table 1 (asterisked) showed an atypical $\delta^{13} \mathrm{C}$ value and a rather high ${ }^{14} \mathrm{C}$ value. This sample was excluded from data evaluation as it was revealed as an outlier by Dixon's $Q$-test. Nevertheless, for 8 out of 9 samples the consensus values for C7 and OxII lie within the 1- $\sigma$ intervals of the measured values.

Table $1{ }^{14} \mathrm{C}$ data of artificial samples. DCAs and HA indicate the presence of dicarboxylic acids (other than oxalic acid) and humic acids, respectively. The specified oxalic acid carbon mass relates to the mass before separation steps. Values and uncertainties were derived from the data reduction after AMS without any correction for a contamination. Average ${ }^{14} \mathrm{C}$ values correspond to the weighted averages of all samples with the uncertainties of the mean. The asterisked sample is considered to be an outlier (see text). Different measurement uncertainties from same sample sizes can be explained by partially shortened measurement times and different $\mathrm{C}^{-}$currents.

\begin{tabular}{llllll}
\hline & $\begin{array}{l}\text { Additional } \\
\text { content }\end{array}$ & $\begin{array}{l}\text { Oxalic acid } \\
\text { starting mass } \\
(\mu \mathrm{g} \mathrm{C})\end{array}$ & $\begin{array}{l}\text { Measured value } \\
(\mathrm{pMC})\end{array}$ & $\begin{array}{l}\delta^{13} \mathrm{C} \\
(\%)\end{array}$ & \begin{tabular}{l} 
Conformity \\
\hline IAEA C7, $\mathrm{SO}_{4}{ }^{2-}$
\end{tabular} \\
& - & 20 & $49.3 \pm 1.2$ & -12.6 & $1 \sigma$ \\
& DCAs & 50 & $50.6 \pm 1.2$ & -10.7 & $1 \sigma$ \\
& DCAs & 20 & $49.7 \pm 2.1$ & -13.5 & $1 \sigma$ \\
& DCAs, HA* & 20 & $47.8 \pm 2.3$ & -18.4 & $1 \sigma$ \\
Average & & & $52.0 \pm 1.8$ & -29.2 & $2 \sigma$ \\
Nominal & & & $49.7 \pm 0.7$ & -13.8 & $1 \sigma$ \\
& & 49.5 & -14.5 & \\
NIST OxII, $\mathrm{SO}_{4}{ }^{2-}$ & & & & \\
& DCAs & 20 & $133.8 \pm 2.6$ & -15.6 & $1 \sigma$ \\
& DCAs, HA & 20 & $132.5 \pm 4.3$ & -16.2 & $1 \sigma$ \\
Average & DCAs, HA & 20 & $131.2 \pm 3.5$ & -16.8 & $1 \sigma$ \\
Nominal & & & $134.1 \pm 3.5$ & -18.6 & $1 \sigma$ \\
& & & $133.1 \pm 1.7$ & -16.8 & $1 \sigma$ \\
\hline
\end{tabular}

All AMS results are based on standard normalization and blank subtraction as described in Wacker et al. (2010). Since the samples are analyzed with a gas ion source where the $\mathrm{CO}_{2}$ feeding rate is kept constant for all samples, there is no significant difference between samples of different sizes for the AMS measurement. Therefore, it is not necessary to take sample size into account for data reduction or interpretation. For corrections of contaminations during the sample preparation, however, the sample size needs to be known. In order to evaluate contaminations during the sample preparation, $\mathrm{C} 7$ and OxII data from Table 1 were considered. The deviations of mean measured values from consensus values are smaller than the $1-\sigma$ uncertainties and are therefore not statistically significant. Thus, no correction for contaminations during sample preparation was applied. An upper limit (2 $\sigma)$ for any possible contamination was determined to be $0.4 \mu \mathrm{g} \mathrm{C}$ assuming a modern contamination. From the very good agreement of mean values with consensus values, we conclude that potential contaminations must be very small and therefore do not pose a problem for small samples of $10 \mu \mathrm{g}$ C. We consider the obtained results as a consequence of avoiding any organic solvent or organic acid for the HPLC eluent preparation. The minimum blank for the entire method corresponds to the blank value for the oxidation in quartz tubes and the ${ }^{14} \mathrm{C}$ measurement and is $0.95 \mathrm{pMC}$ (Fahrni et al. 2010). 
Results of the standard addition of $\mathrm{C} 7$ oxalic acid reference material to the ambient filter from Ispra are shown in Table 2. Since the spiking solution dilutes the sample matrix, this test could reveal insufficient separation and problems related to overloaded analytical columns. The data obtained from the standard addition suggest that high matrix contents of ambient aerosol samples do not impair the separation of oxalic acid from other organic compounds and demonstrate the robustness of our separation method as calculated values coincide with the $1-\sigma$ confidence interval of measured values.

Table 2 Standard addition of the Ispra sample with IAEA C7 oxalic acid. Calculation uncertainties refer to the measurement uncertainty of the pure Ispra sample multiplied by the fraction of Ispra sample for the standard addition. Measurement uncertainties were calculated from counting statistics. For the standard addition samples (asterisked), an additional uncertainty for the sample composition (Gaussian error propagation) was added. The $0 \%$ fraction values of the Ispra sample corresponds to the nominal value of $\mathrm{C} 7$ and the measured value from Table 1, respectively.

\begin{tabular}{clll}
\hline $\begin{array}{l}\text { Fraction of Ispra sample } \\
(\%)\end{array}$ & $\begin{array}{l}\text { Calculated value } \\
(\mathrm{pMC})\end{array}$ & $\begin{array}{l}\text { Measured value } \\
(\mathrm{pMC})\end{array}$ & $\begin{array}{l}\text { Overlap of confidence } \\
\text { intervals }\end{array}$ \\
\hline 100 & & $84.0 \pm 2.3$ & \\
40.1 & $63.4 \pm 0.9$ & $60.9 \pm 2.4^{*}$ & $1 \sigma$ \\
15.1 & $54.7 \pm 0.3$ & $56.2 \pm 1.8^{*}$ & $1 \sigma$ \\
0 & $49.5 \pm 0.1$ & $49.7 \pm 0.7$ & \\
\hline
\end{tabular}

Data obtained from the 2 ambient aerosol filter samples are shown in Table 3. The non-fossil carbon fraction was calculated according to Szidat et al. (2009) using a value of $110.9 \mathrm{pMC}$ as a reference for non-fossil sources originating from biogenic and wood-burning emissions. The non-fossil carbon fractions of $75 \%$ and $83 \%$ reveal mainly non-fossil sources for the oxalic acid formation for both the rural site in the late summer period (Ispra) and the urban site in a late winter period (Gothenburg). A typical sample size of $15 \mu \mathrm{g} \mathrm{C} \mathrm{(56 \mu g} \mathrm{oxalic} \mathrm{acid)} \mathrm{after} \mathrm{water} \mathrm{extraction} \mathrm{corresponds} \mathrm{to}$ about 500 to $1600 \mathrm{~m}^{3}$ of sampled air yielding about $10 \mu \mathrm{g} \mathrm{C}$ for AMS measurements. Other DCAs were not measured due to their low occurrence. Nevertheless, the amounts of succinic, malonic, and maleic acid were found to be sufficient for a ${ }^{14} \mathrm{C}$ measurement of a pooled fraction.

Table 3 Ambient sample data. Oxalic acid content of samples was determined from IC chromatograms.

\begin{tabular}{lllllll}
\hline & $\begin{array}{l}\text { Oxalic acid after } \\
\text { water extraction } \\
(\mu \mathrm{g} \mathrm{C})\end{array}$ & $\begin{array}{l}\text { Oxalic } \\
\text { acid } \\
\left(\mathrm{ng} / \mathrm{m}^{3}\right)\end{array}$ & $\begin{array}{l}\text { OxC/TC } \\
\text { ratio } \\
(\%)\end{array}$ & $\begin{array}{l}\text { Measured } \\
\text { value } \\
(\mathrm{pMC})\end{array}$ & $\begin{array}{l}\text { Non-fossil } \\
\text { fraction } \\
(\%)\end{array}$ & $\begin{array}{l}\delta^{13} \mathrm{C} \\
(\%)\end{array}$ \\
\hline Sample & & & & $81.7 \pm 5.3$ & & -23.2 \\
Ispra-1 & & & & $84.5 \pm 2.5$ & & -16.6 \\
Ispra-2 $(n=2)$ & $36.4 \pm 3.6$ & $162 \pm 16$ & $0.98 \pm 0.09$ & $84.0 \pm 2.3$ & $75 \pm 8$ & -17.2 \\
Gothenburg & $15.4 \pm 1.5$ & $49 \pm 5$ & $0.44 \pm 0.11$ & $92.1 \pm 2.0$ & $83 \pm 9$ & -23.8 \\
\hline
\end{tabular}

\section{CONCLUSION AND FUTURE WORK}

We have developed an efficient method for the separation of oxalic acid from ambient aerosols for ${ }^{14} \mathrm{C}$ measurements on a microgram scale. 2D-chromatography is used for a complete separation of compounds of interest from contaminants. The total efficiency is up to $66 \%$ and no evident contamination was revealed by the data analysis. This method therefore provides high yields at a very low blank level. Even though the method is applicable to many carboxylic acids, the measurement of 
most compounds other than oxalic acid is restricted due to their low abundance in ambient aerosol samples and the requirements of microgram samples for AMS measurements. The first data from 2 ambient samples from Ispra and Gothenburg suggest mainly non-fossil sources for oxalic acid in the atmosphere, but more samples have to be analyzed to gain detailed information on the sources of oxalic acid in ambient aerosols.

Future work will involve CSRA of oxalic acid and compound-class-specific ${ }^{14} \mathrm{C}$ measurements of other DCAs in combination with OC and EC analysis at different sites during different seasons to obtain a more systematic insight into sources of oxalic acid and further DCAs in aerosols.

\section{ACKNOWLEDGMENTS}

We thank J-P Putaud (JRC Ispra), AS Shannigrahi and M Hallquist (University of Gothenburg) for the sampling of aerosols.

\section{REFERENCES}

Fahrni SM, Gäggeler HW, Hajdas I, Ruff M, Szidat S, Wacker L. 2010. Direct measurements of small ${ }^{14} \mathrm{C}$ samples after oxidation in quartz tubes. Nuclear Instruments and Methods in Physics Research B 268(78):787-9.

Fisseha R, Saurer M, Jäggi M, Siegwolf RTW, Dommen J, Szidat S, Samburova V, Baltensperger U. 2009. Determination of primary and secondary sources of organic acids and carbonaceous aerosols using stable carbon isotopes. Atmospheric Environment 43(2): 431-7.

Hori M, Ohta S, Murao N, Yamagata S. 2003. Activation capability of water soluble organic substances as CCN. Aerosol Science 34(4):419-48.

Hsieh L-Y, Kuo S-C, Chen C-L, Tsai YI. 2007. Origin of low-molecular-weight dicarboxylic acids and their concentration and size distribution variation in suburban aerosol. Atmospheric Environment 41(31):664861.

Kawamura K, Ikushima K. 1993. Seasonal changes in the distribution of dicarboxylic acids in the urban atmosphere. Environmental Science and Technology 27(10):2227-35.

Kawamura K, Usukura K. 1993. Distributions of low molecular weight dicarboxylic acids in the north pacific aerosol samples. Journal of Oceanography 49(3):271-83.

Legrand M, Preunkert S, Oliveira T, Pio CA, Hammer S, Gelencsér A, Kasper-Giebl A, Laj P. 2007. Origin of $\mathrm{C}_{2}-\mathrm{C}_{5}$ dicarboxylic acids in the European atmosphere inferred from year-round aerosol study conducted at a west-east transect. Journal of Geophysical Research 112: D23S07, doi:10.1029/2006JD008019.

Limbeck A, Handler M, Neuberger B, Klatzer B, Puxbaum H. 2005. Carbon-specific analysis of humic-like substances in atmospheric aerosol and precipitation samples. Analytical Chemistry 77(22):7288-93.
Mandalakis M, Gustafsson Ö, Alsberg T, Egebäck A-L, Reddy CM, Xu L, Klanova J, Holoubek I, Stephanou EG. 2005. Contribution of biomass burning to atmospheric polycyclic aromatic hydrocarbons at three European background sites. Environmental Science and Technology 39(9):2976-82.

Matsumoto K, Kawamura K, Uchida M, Shibata Y, Yoneda M. 2001. Compound specific radiocarbon and ${ }^{13} \mathrm{C}$ measurements of fatty acids in a continental aerosol sample. Geophysical Research Letters 28(24): 4587-90.

Ruff M, Wacker L, Gäggeler HW, Suter M, Synal H-A, Szidat S. 2007. A gas ion source for radiocarbon measurements at $200 \mathrm{kV}$. Radiocarbon 49(2):307-14.

Ruff M, Szidat S, Gäggeler HW, Suter M, Synal H-A, Wacker L. 2010. Gaseous radiocarbon measurements of small samples. Nuclear Instruments and Methods in Physics Research B 268(7-8):790-4.

Synal H-A, Döbeli M, Jacob S, Stocker M, Suter M. 2004. Radiocarbon AMS towards its low-energy limits. Nuclear Instruments and Methods in Physics Research B 223-224:339-45.

Szidat S, Jenk TM, Gäggeler HW, Synal H-A, Fisseha R, Baltensperger U, Kalberer M, Samburova V, Wacker L, Saurer M, Schwikowski M, Hajdas I. 2004. Source apportionment of aerosols by ${ }^{14} \mathrm{C}$ measurements in different carbonaceous particle fractions. Radiocarbon 46(1):475-84.

Szidat S, Ruff M, Perron N, Wacker L, Synal H-A, Hallquist M, Shannigrahi AS, Yttri KE, Dye C, Simpson D. 2009. Fossil and non-fossil sources of organic carbon (OC) and elemental carbon (EC) in Göteborg, Sweden. Atmospheric Chemistry and Physics 9(5): 1521-35.

Wacker L, Christl M, Synal H-A. 2010. Bats: a new tool for AMS data reduction. Nuclear Instruments and Methods in Physics Research B 268(7-8):976-9. 